Published in final edited form as:

J Cancer Surviv. 2014 September ; 8(3): 427-436. doi:10.1007/s11764-014-0355-5.

\title{
Adopting a survivor identity after cancer in a peer support context
}

\author{
Bronwyn A. Morris, \\ Griffith Health Institute, Griffith University, Building G05 2.44, Brisbane, QLD 4222, Australia. \\ Cancer Council Queensland, Brisbane, Australia
}

\section{Stephen J. Lepore,}

Department of Public Health, Temple University, Philadelphia, USA

\section{Bridget Wilson,}

Griffith Health Institute, Griffith University, Building G05 2.44, Brisbane, QLD 4222, Australia.

Cancer Council Queensland, Brisbane, Australia

\section{Morton A. Lieberman,}

Department of Psychiatry, University of California, San Francisco, USA

Jeff Dunn, and

Cancer Council Queensland, Brisbane, Australia. School of Social Science, University of

Queensland, Brisbane, Australia. School of Public Health, Griffith University, Brisbane, Australia

\section{Suzanne K. Chambers}

Griffith Health Institute, Griffith University, Building G05 2.44, Brisbane, QLD 4222, Australia. Cancer Council Queensland, Brisbane, Australia. Prostate Cancer Foundation Australia, Sydney, Australia. Health \& Wellness Institute, Edith Cowan University, Perth, Australia. Centre for Clinical Research University of Queensland, Brisbane, Australia

\section{Abstract}

Purpose-The term cancer survivor can refer to individuals from diagnosis through the rest of their life. However, not all people with cancer identify as a survivor, and underlying factors and correlates are yet to be well-explored empirically.

Methods-Study 1 surveyed men in a prostate cancer peer support network ( $n=514$ ), exploring psychosocial variables related to adopting a survivor identity. Study 2 interviewed 160 women with breast cancer in an online support group and collected observational data, assessing how survivor identity relates to perceptions of and participation in online support groups.

Results-For men, survivor identity (35\%) was related to lower levels of threat appraisal ( $p=$. 000 ), more deliberate rumination ( $p=.042$ ), gaining greater understanding of cancer experience through peers $(p=.041)$ and a higher, though marginally significant, level of posttraumatic growth $(p=.052)$. Women adopting a survivor identity $(50 \%)$ had higher rates of online support group

Correspondence to: Bronwyn A. Morris

Conflict of interest The authors wish to state that they have no conflicts of interest to declare in regards to this submission to the Journal of Cancer Survivorship. 
posts ( $p=.048$ ), a greater feeling of mattering to the group ( $p=.002)$, rated the group as more helpful ( $p=.004$ to .01 ) and had less difficulty in relating to the group ( $p=.002)$ than women not identifying as a survivor.

Conclusions-Survivor identity was related to active and positive engagement with peers, and cognitive processing.

Implications for cancer survivors-While the cancer survivor metaphor may be salient for some people diagnosed with cancer, many did not associate with the term, highlighting the complexity surrounding survivorship discourse and the need to be sensitive to unique individual needs in psychosocial interventions that involve groups.

\section{Keywords}

Cancer; Survivor identity; Peer support; Posttraumatic growth

\section{Introduction}

Over the past four decades, the language and metaphors used to describe cancer have evolved into a particular lexicon matching social and community aspirations in cancer control [1]. Perhaps the most high-profile early example was the coining of the phrase 'The war against cancer' that emerged with the signing of the National Cancer Act of 1971 by then US President Richard Nixon [2,3]. While this may have been an early herald of a shift from seeing most people with cancer as 'victims' to 'survivors', it was only in 1986 that the National Coalition for Cancer Survivorship (NCCS) was formed as an advocacy group for cancer survivors [4]; a further 10 years to the formation of the Office of Cancer Survivorship by the National Cancer Institute [3]; and yet another 10 years for the founding of this journal, which is dedicated to the growing field of cancer survivorship studies [5]. These groups have generally coalesced around a definition of a cancer survivor as any individual from the time of diagnosis, through the balance of his or her life [6].

However, not all individuals diagnosed with cancer identify as a survivor, with studies showing that endorsement of this term ranges from 26 to $90 \%$ across people diagnosed with breast, prostate and colorectal cancers [7-11]. Qualitative research suggests that for some the term may be unrealistically positive and not reflect the inherent uncertainty and ongoing difficulty individuals' may experience [12,13]. Some people diagnosed with cancer do not wish to be defined by their illness and perceive that the term cancer survivor creates an unwanted focus on their cancer [14]. Diagnosed individuals may also believe that their experience was not severe enough to warrant self-identification as a survivor [12-14]. By contrast, identifying with the term cancer survivor may be empowering as it provides an alternative identity to those stereotypical of illness [15] and a mechanism to replace those identities threatened or lost as a result of the cancer $[9,16]$.

Emerging studies have proposed a number of variables to be associated with whether someone sees themself as a cancer survivor, including sociodemographic and cancer-related variables. For example, individuals who are older in age, with lower education [8] and undergoing chemotherapy $[9,11]$ are more likely to adopt a survivor identity. Individuals 
who believe their prognosis is good compared to others and that their treatment is curative are also more likely to endorse a cancer survivor identity [11]. Identifying as a cancer survivor has been linked to several psychological outcomes including better mental health [9, 11], positive affect [7], satisfaction with life, decreased somatization [10] and higher selfesteem. In addition to being related to better psychological adjustment, survivor identity has also been found to be associated with benefit finding and perceiving positive life change after cancer $[8,10]$. Positive life changes, or posttraumatic growth (PTG), are commonly reported after a diagnosis of cancer [17]. PTG can occur as the individual experiences cancer-related rumination and examines core beliefs [18], which are factors that may be related to changes in self-identity after cancer and perceiving oneself to be a survivor.

The formation of identity may also be based upon available discourses and relationships [19]. Specifically, peer support groups have been shown to positively influence perceptions of self and identity following cancer by assisting in meaning-making of the cancer experience [20-22]. Following the participation in a challenge-based peer support event, a study of women diagnosed with breast cancer showed that some participants attached new positive meaning to the term breast cancer survivor and identified as a survivor [16]. Also, engagement in cancer-related activities, such as talking about one's cancer experience and prevention, as well as the use of more instrumental and active coping have also been found to be associated with endorsement of the survivor label [8]. On this view, we propose that individuals may be more likely to self-identify as a cancer survivor when there is a sense of connection or active engagement with peers. A peer support network may allow survivors to reduce feelings of alienation and form a new sense of identity in this period of postdiagnosis adjustment.

Alternatively, individuals who do not adopt a survivor identity may be disinclined to associate with other cancer survivors or, should they decide to join a cancer support group, they might find it difficult to relate to and engage with other patients who have adopted a survivor identity. Because people adopting the survivor identity tend to have more positive psychological outcomes [7-11], they may present themselves in groups as being resilient and optimistic. This could potentially alienate individuals who feel vulnerable and uncertain or anxious about what the future holds. To the extent that persons reject the survivor identity, they may not have their needs met in a support group, may be reluctant to express their true feelings to the group — especially feelings of vulnerability — and may find it difficult to relate to other group members. Indeed, one qualitative study showed that resisting or giving up a cancer identity is one reason why people leave cancer support groups [23]. In contrast, individuals who come to a group with a survivor identity and find others who share this identity will quickly have a sense of belonging and likely be eager to engage with other members [16]. By studying how survivor identity might influence patients' perceptions of and participation in cancer support groups, we may be able to improve interventions to address the broad range of unmet needs of diverse patient populations.

This paper presents two studies assessing factors associated with identifying as a cancer survivor. These studies were conducted with people involved in peer support as this context can be an important part of the post-diagnosis experience for people diagnosed with cancer. As there appear to be many influencing factors that determine survivor identification and 
there is a diverse range of prevalence rates in the few existing studies, we did not

hypothesize a prevalence rate for survivorship identification in the two current studies.

\section{Study 1 hypotheses}

Study 1 focused on identifying psychosocial factors that might contribute to the adoption of a cancer survivor identity. It was anticipated that greater levels of threat appraisal, examination of core beliefs, rumination, peer support factors (gaining an understanding of the cancer experience through peers and a sense of connection to peers) and posttraumatic growth would be related to an endorsement of a cancer survivor identity.

\section{Study 2 hypotheses}

Study 2 focused on describing how individual differences in adoption of a cancer survivor identity might influence experiences and behaviours in an online breast cancer support group. It was anticipated that support group members who adopted a cancer survivor identity might exhibit relatively high engagement in the group, as measured by attendance and amount of posting in the groups, relative to members without a survivor identity. Furthermore, we anticipated that those members with a survivor identity would have more positive appraisals about the helpfulness of the group experience and find it easier to relate to other group members. In addition, this study allowed us to explore associations between demographic and medical factors and survivor identity that have been reported in other studies.

\section{Method-study 1}

\section{Participants and procedure}

Ethical approval to conduct this study was granted by the human research ethics committee of Griffith University (PSY/35/12/HREC). Letters of invitation were sent to all members ( $n=973$ ) of the Brisbane Prostate Support Network (BPSN), which is affiliated with the Prostate Cancer Foundation of Australia and Cancer Council Queensland. The BPSN is a network of men diagnosed with prostate cancer who receive a monthly newsletter. BPSN holds regular monthly support group meetings; however, not all members attend the meetings. All members were posted the study material and 514 surveys were returned to the research team (52.8\% response). Participants were 70 years of age ( $\mathrm{SD}=8.36$, range 44-94), and on average, it had been 7.50 years $(\mathrm{SD}=4.66)$ since their diagnosis of prostate cancer. Participants were generally married or in a relationship (84.2\%) and were retired (67.1\%). Participants predominantly had an education level higher than school (trade or technical college or diploma $32.7 \%$; university or college degree $36.0 \%$ ). The most common forms of prostate cancer treatment received by the participants included radical prostatectomies $(57.2 \%)$, external beam radiation $(32.9 \%)$ and hormone therapy $(23.5 \%)$.

\section{Measures}

Cancer identity-Participants' endorsement of cancer identity was assessed using a single-item question, 'When you think about yourself in relation to your cancer, which of the following phrases best describes you?' [7]. Respondents were asked to only endorse one of the five possible labels: I am a cancer patient, I am a cancer survivor, I am a cancer victim, I 
am a person who has had (or has) cancer, or other. Previous research has successfully utilised this single-item question [10].

Threat appraisal-The threat subscale of a revised version of the Stress Appraisal Measure (SAM) [24] was used to assess the level of perceived threat participants associated with their cancer experience. Participants were instructed 'As you rate the following questions, we would like you to consider how you feel when thinking about your prostate cancer' and rated the items on a five-point Likert scale from 1 (not at all), 3 (moderately), to 5 (extremely). Higher mean scores indicated greater optimism and self-efficacious thoughts (e.g. 'I perceive this situation as threatening') and internal consistency was high $(a=.85)$.

Peer support factors-Phinney and Ong's [25] Multigroup Ethnic Identity MeasureRevised (MEIM-R) was adapted to reflect participant's level of connection to peers (e.g. 'I feel a strong connection to other men with prostate cancer') and whether participants sought an understanding of their cancer through peers (e.g. 'I have spent time trying to find out more about other men with prostate cancer'). Six items were rated on a five-point Likert scale from 1 (strongly disagree) to 5 (strongly agree). Higher mean scores indicated a greater level of connection or seeking understanding of cancer through peers and internal consistency for each subscale was high ( $a=.80$ and .76 , respectively).

Core beliefs-The Core Beliefs Inventory [26] assessed the degree to which an individual's core beliefs were examined after being diagnosed with cancer. Participants rated nine items (e.g. 'Because of the event, I seriously examined my beliefs about the meaning of my life') on a six-point Likert scale ranging from 0 (not at all) to 5 (to a very great degree). Higher mean scores indicated a greater degree of examining core beliefs as a result of their prostate cancer and internal consistency was high $(a=.92)$.

Rumination-The Event-Related Rumination Inventory (ERRI) [27] was used to measure intrusive and deliberate forms of rumination. Participants rated how often they experienced each of the 20 items on a 4-point Likert scale ranging from 0 (not at all) to 3 (often).

Example items included 'I could not keep images or thoughts about the event from entering my mind' (intrusive rumination) and 'I forced myself to think about my feeling about my experience' (deliberate rumination). Higher summed scores on each subscale indicated greater levels of intrusive and deliberate rumination. High internal consistency was found for both the intrusive and deliberate subscales ( $a=.96$ and .90 , respectively).

Posttraumatic growth-Perceived positive life change that had occurred as a result of struggling with cancer was measured using the Posttraumatic Growth Inventory (PTGI) [28]. A 6-point Likert scale ranging from 0 (not at all) to 5 (to a very great degree) was used to rate 21 items (e.g. 'My priorities about what is important in life' and 'Putting effort into my relationships'). Higher summed scores indicated greater perceived growth and high internal consistency was found $(a=.95)$. 


\section{Statistical analyses}

All continuous variables were checked for normality by generating skewness statistics and visual inspection of histograms. Time since diagnosis and the variable related to seeking an understanding of cancer through peers remained highly skewed after log transformations were applied. Threat appraisal was also skewed and analyses were run prior to and after a $\log 10$ transformation was applied, with no change in significance of results. Therefore, the untransformed variables were used in analyses for ease of interpretability of these three variables [29]. Prior to conducting regression analyses, no evidence of multicollinearity was found between variables, with bivariate correlations not exceeding the threshold of .8 [30]. A strong correlation was evident between intrusive rumination and core beliefs variables ( $r=$ $621)$, as well as intrusive rumination and deliberate rumination variables ( $r=635)$. The two subscales of the peer support measure (connection to peers and seeking an understanding of cancer through peers) were also strongly correlated ( $r=.744)$. Core beliefs was strongly correlated with PTG ( $r=.608)$. These correlations emphasise that while these constructs are strongly related they are still distinct [30]. Bivariate intercorrelations between variables are presented in Table 1.

The differences in psychosocial variables between individuals who possess a survivor identity and those who self-identify otherwise were examined using $t$ tests for continuous variables and chi-square tests for categorical sociodemographic variables. Stepwise logistic regression was used to assess the model of variables that was related to identifying as a cancer survivor. The variables entered into the regression analyses were determined a priori as per theoretical consideration, including controlling for sociodemographic and cancerrelated variables in step one of the model.

\section{Results-study 1}

\section{Cancer survivor identity}

Participants preferred to see themselves in the following way: a person who has/had cancer (53.1\%), a cancer survivor (35.0\%), a cancer patient $(6.2 \%)$, a victim $(1.9 \%)$ and other (2.1\%) (1.7\% were missing values). No respondent who identified as other described an alternative identity. For subsequent analyses, the data was recoded to classify respondents as either someone who identified as a survivor $(n=180)$ or someone who did not $(n=326)$.

\section{Variables related to cancer survivor identity}

Respondents who did and did not identify as a cancer survivor differed significantly on psychosocial variables (see Table 2). No differences were evident between the two groups on sociodemographic and cancer-related variables including age $(p=.761)$, relationship status ( $p=.883$ ), education $(p=.449)$ and time since diagnosis $(p=.098)$.

Stepwise logistic regression models were conducted to assess the psychosocial variables that were related to a cancer survivor identity. Age, relationship status, education and time since diagnosis were entered in block one, and this model was not significant, $\chi^{2}(7)=6.16, p=.521$, explaining 1.4-2.0\% (Cox and Snell-Nagelkerke $R^{2}$ ) of the variance. Threat appraisal, examining core beliefs, intrusive rumination, deliberate rumination, understanding through 
peers, connection to peers and PTG were entered in the second block, and this model was significant, $\chi^{2}(14)=40.15, p=.000$, explaining 10.3-14.1\% (Cox and Snell-Nagelkerke $R^{2}$ ) of the variance. Lower threat appraisal, higher deliberate rumination and gaining an understanding through peers were significantly associated with a cancer survivor identity (Table 3). A trend was evident for higher levels of PTG to be associated with survivor identity $(p=.052)$.

\section{Method-study 2}

\section{Participants and procedure}

Ethical approval to conduct this study was obtained from the institutional review board of Temple University (protocol number 13705). Participants were part of a larger randomized controlled trial comparing the efficacy of two online support groups (OSGs) designed to reduce psychological distress among women who had elevated distress. Eligible participants were within 3 years of being treated for stage 1 or 2 breast cancer, and were preselected on the basis of elevated levels of anxiety or depression symptoms as measured by the Hospital Anxiety and Depression Scales [31]. Details of the trial methodology can be found in a published protocol paper [32].

Twelve OSGs were conducted with approximately 14 women per group. Groups were manualized and lasted for 6 weeks. Six groups were randomized to a standard online support group (S-OSG) and six to an enhanced, prosocial online support group (P-OSG). The SOSG and P-OSG shared many common features, including an asynchronous discussion board that participants could use to post messages or read posts from other group members at any time during the intervention period and a weekly 90-min live online chat room in which participants discussed topics relevant to breast cancer (e.g. body image, fear of recurrence and lymphedema). Trained and supervised professionals facilitated the groups. The S-OSG condition was modelled after empirically validated OSGs [33, 34] and emphasized supportive-expressive exchanges among group members. The P-OSG intervention had enhancements that were designed to increase the exchange of helping behaviours.

Multiple methods of data collection were used. Self-report data were collected via structured telephone interviews administered approximately 1 month before and after the intervention. Observational data, such as attendance and number of posts, were derived from the analyses of transcripts of the discussion board and chat room interactions. Medical data were obtained from tumour registry databases. Because this study investigated how survivor identity relates to OSG behaviours and perceptions, only data from those participants who completed the post-intervention survey and answered the items about survivor identity and OSG experiences were analyzed. This resulted in a sample of 160 participants (out of 183 randomized).

Participants were, on average, 52 years of age ( $\mathrm{SD}=7.6$, range 26-65), predominantly Caucasian (96\%) and married (86\%). They had a high level of education attainment (19\% high school graduate only; $31 \%$ some college; $27 \%$ college degree; $23 \%$ advance professional or graduate degree). Slightly more women had stage 1 (56\%) than stage 2 
(44\%) breast cancer, and the majority had received adjuvant chemotherapy $(64.4 \%)$. Average time since diagnosis ranged from 6 to 36 months ( $M=24$ months, $\mathrm{SD}=7.1$ ).

\section{Measures}

Cancer identity-As in study 1, participants' endorsement of cancer identity was assessed using a single-item question, 'When you think about yourself in relation to your cancer, which of the following phrases best describes you?' [7]. Respondents were asked to only endorse one of the five possible labels: I am a cancer patient I am a cancer survivor, I am a cancer victim, I am a person who has had (or has) cancer or other.

Mattering to others in the online support group-The General Mattering Scale [35] was adapted to assess the degree to which participants felt that they mattered to members of the OSG (e.g. 'How important were you to others in the group'). Participants rated five items on a five-point Likert scale from 0 (not at all) to 4 (very much). The measure is valid and reliable $[35,36]$ and high internal consistency was found in this sample $(a=.93)$.

Level of participation-Level of participation was measured by counting the number of times a participant posted a message on the asynchronous discussion board and in the weekly synchronous chat sessions. In addition, the number of chat sessions attended and the number of weeks in which participants visited the discussion board were counted.

Helpful group experiences-The Helpful Group Experiences [HGE; 37-39] questionnaire assessed participants' perceptions of the importance of different group experiences in helping them and addressing the problems for which OSGs are designed to address. The measure was adapted for the larger trial by adding questions related to the importance of providing help to others. The HGE captured interpersonal factors (e.g. group belonging, support, guidance and altruism) and intrapersonal factors (e.g. insight and selfdisclosure) presumed to fuel therapeutic benefits in groups [38]. Participants responded to the 21-item measure by rating the importance on a 5-point Likert scale from 0 (not at all) to 4 (very much). The following five HGE subscales all had acceptable internal consistency: emotional and belonging support (e.g. experienced a sense of belonging, got support and encouragement; $a=.88$ ), cognitive growth and informational (e.g. gained access to important information, got new understandings or explanations; $a=.89$ ), disclosure (e.g. expressed my true feelings, talked about fears of recurrence; $a=.71$ ), existential concerns (e.g. deepened my spiritual life, confronted difficult problems and fears; $a=.76)$ and altruism (e.g. I offered empathy or compassion, I gave emotional support and encouragement; $a=93$ ).

Perceived difficulties in relating to the group-Two questions also assessed the degree to which participants perceived difficulties in relating to the group: 'It was difficult relating to other women in the group' and 'I felt I had nothing to offer'. Participants responded to each question on a five-point Likert scale from 0 (not at all) to 4 (very much) and a mean rating of the two items was assessed. Internal consistency was adequate $(a=.69)$. 


\section{Statistical analyses}

As in study 1, we first assessed normality of the distributions of the variables. The number of posts (participation level) had a moderate skew, but other continuous variables were normally distributed. A square-root transformation effectively normalized the skewed data. $T$ tests were used to investigate survivor identity (yes/no) group differences in continuous variables. Chi-square techniques were used to examine the relation of survivor identity to categorical demographic characteristics (marital status and education background) and medical characteristics (stage of breast cancer and receipt of chemotherapy).

\section{Results-study 2}

\section{Cancer survivor identity}

Participants preferred to see themselves in the following way: a cancer survivor (44\%), a person who has/had cancer (42\%), a cancer patient (6\%), a cancer victim (2\%) and other (6\%). Of the nine participants who selected 'other', six said 'cancer warrior' and three said 'sur-thriver'. These identity labels emerged in the OSG chat rooms. For purposes of analysis, these participants were categorized as adopting a survivor identity, bringing the number categorized as 'survivor identity' to $n=80$. The other categories were collapsed into the 'no survivor identity' category $(n=80)$.

\section{Relation of survivor identity to demographic and medical variables}

Adopting a survivor identity was more common among participants with less than a college degree $(60 \%)$ than among women with a college degree $(40 \%), \chi^{2}(3)=9.52, p=.023$.

Adopting a survivor identity was also more common among those who had chemotherapy $(74 \%)$ than those who did not $(26 \%), \chi^{2}(1)=6.13, p=.013$. Survivor identity was unrelated to age, stage of cancer or time since diagnosis.

\section{Relation of survivor identity to attendance and posting frequency}

As shown in Table 4, participants attended approximately four $(67 \%)$ of the six chat sessions and visited the discussion board approximately $3(50 \%)$ of the 6 weeks. Survivor identity was unrelated to attendance. However, participants adopting a survivor identity were significantly more active posters in chat than their counterparts who did not adopt a survivor identity. Similar group differences were observed for level of discussion board posting, but the effect was marginally significant. The group differences in posting behaviour were the same for normalized and raw data. Thus, lurking behaviour was more common among those with a non-survivor identity.

\section{Relation of survivor identity to perceived relatedness to the group}

As shown in Table 4, participants with a survivor identity rated how much they mattered to the group at a significantly higher level than their counterparts without a survivor identity. The non-survivor group rated their level of difficulty in relating to the group significantly higher than the survivor group. 


\section{Survivors and non-survivors perceptions of helpful group experiences}

Participants rated all of the helpful group experiences as moderately important. The highest overall mean was for disclosure (2.61, SD=.98) and the lowest was for existential concerns (2.04, $\mathrm{SD}=1.02)$. As shown in Table 4, there were significant differences between the survivor and non-survivor identity groups on all but one (disclosure) of the helpful group experience measures. Relative to the non-survivor identity group, the survivor identity group rated each of the following helpful group experiences as significantly more important: emotional belonging and support, cognitive growth and information, existential concerns and altruism.

\section{Discussion}

The current studies explored potential variables that were related to identifying as a cancer survivor and results across both studies showed that endorsing this term was associated with positive appraisals of and more active behaviour in peer support groups. Survivor identity was not related to sociodemographic or cancer-related variables in study 1 . However, similar to previous studies, women in study 2 who had less than a college degree [8] were more likely to identify as a survivor. Study 2 also replicated a previously found association between adopting a survivor identity and having received chemotherapy as treatment $[9,11]$. The men diagnosed with prostate cancer who felt that they gained an understanding of their cancer experience through peers were likely to identify as a cancer survivor. Compared to non-survivors, women diagnosed with breast cancer who identified as a cancer survivor were likely to be more active in the online chat room, felt that they mattered to the group, had less difficulty relating to the group and rated group experiences as more helpful. For the cancer survivor, identity may be the product of an interaction between perception of self and interactions with other individuals and their social environment [40]. This social interaction is particularly salient in this context as belonging to a peer network can reduce feelings of isolation, promote optimism and enhance active coping strategies to deal with the cancer experience [19, 20, 41, 42].

However, results from study 2 also suggest that individuals who are resistant to identifying as a cancer survivor might not fully engage in or perceive benefits from support groups in which the survivor identity is salient among group members. Over time, such groups are likely to become increasingly homogenous, as individuals who share the survivor identity stay engaged and mutually reinforce the survivor identity and those who do not share this identity disengage and possibly abandon the group. Within professionally facilitated support groups, some of these dynamics can be regulated to some extent if facilitators are aware that they are happening. It is likely, however, that tempering group members' enthusiasm about a shared survivor identity would be difficult. Anecdotally, it was evident in the online support group transcripts from study 2 that some groups were very enthusiastic about the survivor identity, referring to one another as fellow 'sur-thrivers' and 'warrior sisters'. The vast majority of online support groups are not professionally facilitated, so these kinds of dynamics will often go unchecked, making it difficult for some people to find a group that satisfies their supportive care needs. 
In addition to peer support factors, men who reported lower threat appraisal and greater deliberate rumination were more likely to see themselves as a cancer survivor. It may be that cancer survivors who belong within a peer support network continue to reflect and ruminate on their cancer, however, at the same time do not appraise their cancer experience as threatening. Perhaps this process underpins the link between cancer survivor identity and PTG, which is consistent with previous research and shown with borderline significance in the present study $[8,10]$. Identifying as a survivor may fit within the theoretical model of PTG as a component of the complex narrative gained from personal growth after a traumatic experience [18]. This complex narrative and changing discourse that emerges for the person diagnosed with cancer can extend well beyond the period of illness and is influenced by social trends $[43,44]$. Further research can explore the how social processes over time can influence the meaning that someone attaches to the term cancer survivor.

The concept of the cancer survivor might be usefully seen as a metaphor for the experience of cancer. Metaphors are a characteristic of language that define the structure of how we see and interact with the world [45]. 'Cancer is War' may be a conceptual metaphor, where cancer is seen as a battle in which there are victims and survivors [1]. This metaphor may explain why women are more likely to endorse a survivor identity when they had undergone chemotherapy as treatment for their breast cancer. Perhaps women who have endured the rigours of chemotherapy feel that they can claim survivorship status [11] as their experience has been severe enough to warrant this title [12-14]. Metaphors and the new discourse surrounding someone diagnosed with cancer can have a powerful influence on the way people behave and understand their cancer experience [1].

While the results from the two current studies suggest that the survivor metaphor has a strong resonance with many men and women in cancer peer support networks, this is not universal. Over a third of men with prostate cancer in study 1 perceived themselves as cancer survivors, while the majority identified as someone who has/had cancer. The prevalence of survivor identity found in study 1 is comparable to another study of men with prostate cancer identifying as a cancer survivor (26\%) [7] and may also be an artefact of the extended length of time since diagnosis in this participant group. Half the women with breast cancer in study 2 perceived themselves as cancer survivors, which is lower than another study with breast cancer survivors (78 \%) [11]. The Jagielski et al. [11] study assessed survivor identity with a dichotomous question ('Do you consider yourself to be a survivor of breast cancer?') and did not give the varied response options offered in study 2 . Furthermore, participants in study 2 were preselected for elevated distress, which may have influenced the likelihood of endorsing a survivor identity as studies have shown positive links between survivor identity and improved psychological outcomes [7, 9-11].

In addition, most participants in study 2 had attained some level of college education, which could partly explain the relatively small proportion of women endorsing the survivor identity. As previously noted, higher education status is associated with a lower probability of adopting a survivor identity. It is possible that this reflects social class and a tendency among middle- and upper-middle class groups to place a higher value on independence and autonomy than lower-class groups, particularly in the US [46]. Perhaps individuals who value independence might be resistant to identifying with a group, and possibly resistant to 
seeking help from or identifying with a peer group. It may be that the needs of people with different education levels should be considered when designing supportive care programs in order to provide care that is tailored and beneficial for particular groups. Along these lines, in several studies with men with prostate cancer, educational attainment has been found to moderate the effectiveness of psychosocial interventions, and although the reasons for this are unclear, this does support the importance of considering both class and context in how people respond to and cope with a diagnosis of cancer [47-49].

Approximately $65 \%$ of men diagnosed with prostate cancer and $55 \%$ of women diagnosed with breast cancer did not perceive themselves as cancer survivors. This result seems contradictory as participants in both studies belonged to a peer support network, and these men and women are likely to be involved in activities that have a personal affiliation with cancer. Of course, as study 2 showed, the degree of engagement in and 'relatedness' to the group was lowest among those who did not adopt a survivor identity. It may also be the case that measuring identity after cancer as dichotomous - that is either a cancer survivor or not -is simplistic and fails to capture the complexity of defining the self after cancer [12]. Studies to date have used measures of identity that are centred around cancer, without taking into account the complex nature of self identity that extends beyond illness. A future research question may be how to expand the metaphor of cancer to be inclusive of precancer experiences and roles, rather than an identity that is centred on being diagnosed with a disease.

While the presented studies provided an exploration of the prevalence and correlates of a survivor identity, this was limited by cross-sectional design such that causality cannot be inferred. It is likely that the significant relationships shown in the current studies are reciprocal. For example, it may be that adopting a survivor identity facilitated greater participation by women in the online support groups. Conversely, these results may also indicate that greater participation in the group enhanced the likelihood of perceiving oneself as a survivor. Further studies incorporating longitudinal design can tease out the nuances of these variables over time. Also, both studies utilised selected samples of peer support network members, limiting generalisability of the findings beyond this participant group. In addition, while a trend was evident between PTG and survivor identity in study 1, the lack of statistical significance may be due to the length of time since diagnosis. On average, men were over 7 years post-diagnosis, which may lessen the salience of factors such as examining core beliefs, intrusive rumination and positive life change as a result of the cancer experience. Strengths of the current studies included high recruitment rates, assessment of two prevalent diagnostic groups and exploration of a number of correlating factors, including cognitions and behaviours, associated with adopting a survivor identity.

\section{Conclusion}

Endorsing a survivor identity was related to active engagement and positive perception of peer support networks, in addition to low threat appraisal, more deliberate rumination about cancer and posttraumatic growth. These results highlight the cognitive and social processes associated with survivor identity. Exploring the salience and correlating factors associated with self-identifying as a cancer survivor helps us to understand the complexities of 
discourse surrounding survivorship. This discourse has evolved over the last few decades, as survival rates have improved and advocacy for support and awareness has become prominent, particularly for breast and prostate cancer. While the cancer survivor metaphor may be salient for some men diagnosed with prostate cancer and some women diagnosed with breast cancer, for a large proportion of others, it may not reflect the language they use to describe themselves and their experience of cancer. The results of these studies have potential implications for providing supportive care services that are cognisant of the potential impact of metaphors in the cancer experience and are sensitive to the needs of persons that may not identify with the survivor identity.

\section{Acknowledgments}

The authors wish to thank the Brisbane Prostate Support Network, the Prostate Cancer Foundation of Australia, Cancer Council Queensland and the National Cancer Institute of the National Institute of Health (Grant CA15887) for their support and assistance in this project. Prof. Suzanne Chambers is supported by an Australian Research Council Future Fellowship.

\section{References}

1. Reisfield GM, Wilson GR. Use of metaphor in the discourse on cancer. J Clin Oncol. 2004; 22(19): 4024-7. [PubMed: 15459229]

2. National Cancer Institute. Milestone (1971): President Nixon declares war on cancer. [cited 2013 22nd April]; Available from: http://dtp.nci.nih.gov/timeline/noflash/milestones/M4_Nixon.htm

3. National Cancer Institute. About cancer survivorship research: History. 2012. [cited 2013 22nd April]; Available from: http://dccps.nci.nih.gov/ocs/history.html

4. National Coalition for Cancer Survivorship. Our History. 2013. [cited 2013 30th April]; Available from: http://www.canceradvocacy.org/about-us/our-history/

5. Feuerstein M. Optimizing cancer survivorship. J Cancer Surviv. 2007; 1:1-4. [PubMed: 18648938]

6. National Cancer Institute. About survivorship research: Survivorship definitions. 2012. [cited 2013 22nd April]; Available from: http://dccps.nci.nih.gov/ocs/definitions.html

7. Bellizzi KM, Blank TO. Cancer-related identity and positive affect in survivors of prostate cancer. J Cancer Surviv. 2007; 1:44-8. [PubMed: 18648944]

8. Park CL, Zlateva I, Blank TO. Self-identity after cancer: "Survivor", “victim”, “patient”, and "person with cancer". J Gen Intern Med. 2009; 24(Suppl 2):430-5. [PubMed: 19096898]

9. Deimling GT, Bowman KF, Wagner LJ. Cancer survivorship and identity among long-term survivors. Cancer Investig. 2007; 25:758-65. [PubMed: 17952742]

10. Chambers SK, et al. Survivor identity after colorectal cancer: antecedents, prevalence and outcomes. Psycho-Oncology. 2012; 21(9):962-9. [PubMed: 21608073]

11. Jagielski $\mathrm{CH}$, et al. A phoenix rising: who considers herself a "survivor" after a diagnosis of breast cancer? J Cancer Surviv. 2012; 6:451-7. [PubMed: 22936393]

12. Kaiser K. The meaning of the survivor identity for women with breast cancer. Soc Sci Med. 2008; 67:79-87. [PubMed: 18450347]

13. Khan NF, et al. Interpretation and acceptance of the term 'cancer survivor': a United Kingdombased qualitative study. Eur J Cancer Care. 2012; 21:177-86.

14. McGrath P, Holewa H. What does the term 'survivor' mean to individuals diagnosed with a haematological malignancy? Findings from Australia. Support Care Cancer. 2012; 20:3287-95. [PubMed: 22549505]

15. Sontag, S. Illness as metaphor. New York: Farrar, Straus, Giroux; 1978.

16. Morris BA, Campbell M, Dwyer M, Dunn J, Chambers SK. Survivor identity and post-traumatic growth after participating in challenge-based peer support programmes. Br J Health Psychol. 2011; 16:660-74. [PubMed: 21199541] 
17. Stanton, AL., Bower, JE., Low, CA. Posttraumatic growth model: sociocultural considerations. In: Calhoun, LG., et al., editors. Handbook of posttraumatic growth: research and practice. New Jersey: Lawrence Erlbaum Associates; 2006. p. 138-75.

18. Calhoun, LG., Tedeschi, RG. Posttraumatic growth in clinical practice. New York: Routledge; 2013.

19. Davies, B., Harre, R. Positioning and personhood. In: Harre, R., van Langenhove, L., editors. Positioning theory. Oxford: Blackwell; 1998.

20. Sabiston CM, McDonough MH, Crocker PRE. Psychological experiences of breast cancer survivors involved in dragon boat program: exploring links to positive psychological growth. J Sport Exerc Psychol. 2007; 29:419-38. [PubMed: 17968046]

21. Burke P. Identity processes and social stress. Am Sociol Rev. 1991; 56:836-49.

22. Lepore, SJ., Kernan, W. Positive life change and the social context of illness: an expanded socialcognitive processing model. In: Park, CL., et al., editors. Medical illness and positive life change: can crisis lead to personal transformation?. Washington, DC: American Psychological Association; 2009. p. 139-52.

23. Ussher JM, et al. A qualitative analysis of reasons for leaving, or not attending, a cancer support group. Soc Work Health Care. 2008; 47(1):14-29. [PubMed: 18956510]

24. Roesch SC, Rowley AA. Evaluating and developing a multidimensional, dispositional measure of appraisal. J Pers Assess. 2005; 85(2):188-96. [PubMed: 16171419]

25. Phinney JS, Ong AD. Conceptualisation and measurement of ethnic identity: current status and future direction. J Couns Psychol. 2007; 54(3):271-81.

26. Cann A, et al. The core beliefs inventory: a brief measure of disruption in the assumptive world. Anxiety, Stress Coping. 2010; 23:19-34. [PubMed: 19326274]

27. Cann A, et al. Assessing posttraumatic cognitive activity: the event related rumination inventory. Anxiety, Stress Coping. 2011; 24(2):137-56. [PubMed: 21082446]

28. Tedeschi RG, Calhoun LG. The posttraumatic growth inventory: measuring the positive legace of trauma. J Trauma Stress. 1996; 9:455-71. [PubMed: 8827649]

29. Tabachnick, BG., Fidell, LS. Using multivariate statistics. 6. Boston: Pearson; 2012.

30. Cohen, J. Statistical power analysis for the behavioural sciences. Hillsdale, NJ: Erlbaum; 1988.

31. Zigmond AS, Snaith RP. The hospital anxiety and depression scale. Acta Psychiatr Scand. 1983; 67:361-70. [PubMed: 6880820]

32. Lepore SJ, et al. Standard versus prosocial online support groups for distressed breast cancer survivors: a randomized controlled trial. BMC Cancer. 2011; 11:379-86. [PubMed: 21867502]

33. Winzelberg AJ, et al. Evaluation of an internet support group for women with primary breast cancer. Cancer. 2003; 97(5):1164-73. [PubMed: 12599221]

34. Lieberman MA, et al. Electronic support groups for breast carcinoma: a clinical trial of effectiveness. Cancer. 2003; 97(4):920-5. [PubMed: 12569591]

35. Marcus, FM. Mattering: its measurement and theoretical significance for social psychology. Paper presented in Annual meeting of the Eastern Sociological Association; Cincinnati: OH. 1991.

36. Rosenberg M, McCullough BC. Mattering: inferred significance and mental health among adolescents. Res Community Ment Health. 1981; 2:163-82.

37. Lieberman MA. Understanding how groups work: a study of homogenous peer group failures. Int J Group Psychother. 1990; 40:31-52. [PubMed: 2318555]

38. Lieberman MA, Golant M. Leader behaviors as perceived by cancer patients in professionally directed support groups and outcomes. Group Dynamics: Theory, Research, and Practice. 2002; 6:267-76.

39. Lieberman, MA., Yalom, ID., Miles, M. Encounter groups: first facts. New York: Basic Books; 1973.

40. Zebrack B. Cancer survivors and quality of life: a critical review of the literature. Oncol Nurse Forum. 2000; 27:1395-401.

41. Lepore SJ, Revenson RA. Social constraints on disclosure and adjustment to cancer. Soc Personal Psychol Compass. 2007; 1(1):313-33. 
42. Lepore, SJ. A social-cognitive processing model of emotional adjustment to cancer. In: Baum, A., Andersen, B., editors. Psychosocial interventions for cancer. Washington, DC: American Psychological Association; 2001. p. 99-118.

43. Harwood J, Sparks L. Social identity and health: an intergroup communication approach to cancer. Health Commun. 2003; 15:145-59. [PubMed: 12742766]

44. Zebrack BJ. Cancer survivor identity and quality of life. Cancer Pract. 2000; 8:238-42. [PubMed: 11898236]

45. Lakoff, G., Johnsen, M. Metaphors we live by. London: The University of Chicago Press; 2003.

46. Kagitcibasi C. Autonomy and relatedness in cultural context: implications for self and family. $\mathrm{J}$ Cross-Cult Psychol. 2005; 36:403-22.

47. Chambers SK, et al. Intervening to improve psychological outcomes for men with prostate cancer. Psycho-Oncology. 2013; 22(5):1025-34. [PubMed: 22549800]

48. Lepore SJ, et al. Improving quality of life in men with prostate cancer: a randomized controlled trial of group education interventions. Health Psychol. 2003; 22(5):443-52. [PubMed: 14570527]

49. Mayer, KU. The sociology of life course and lifespan psychology: diverging or convering pathways?. In: Stauginger, UM., Lindenberger, U., editors. Understanding human development: dialogues with lifespan psychology. Dordrecht: Kluwer Academic Publishers; 2003. p. 463-81. 


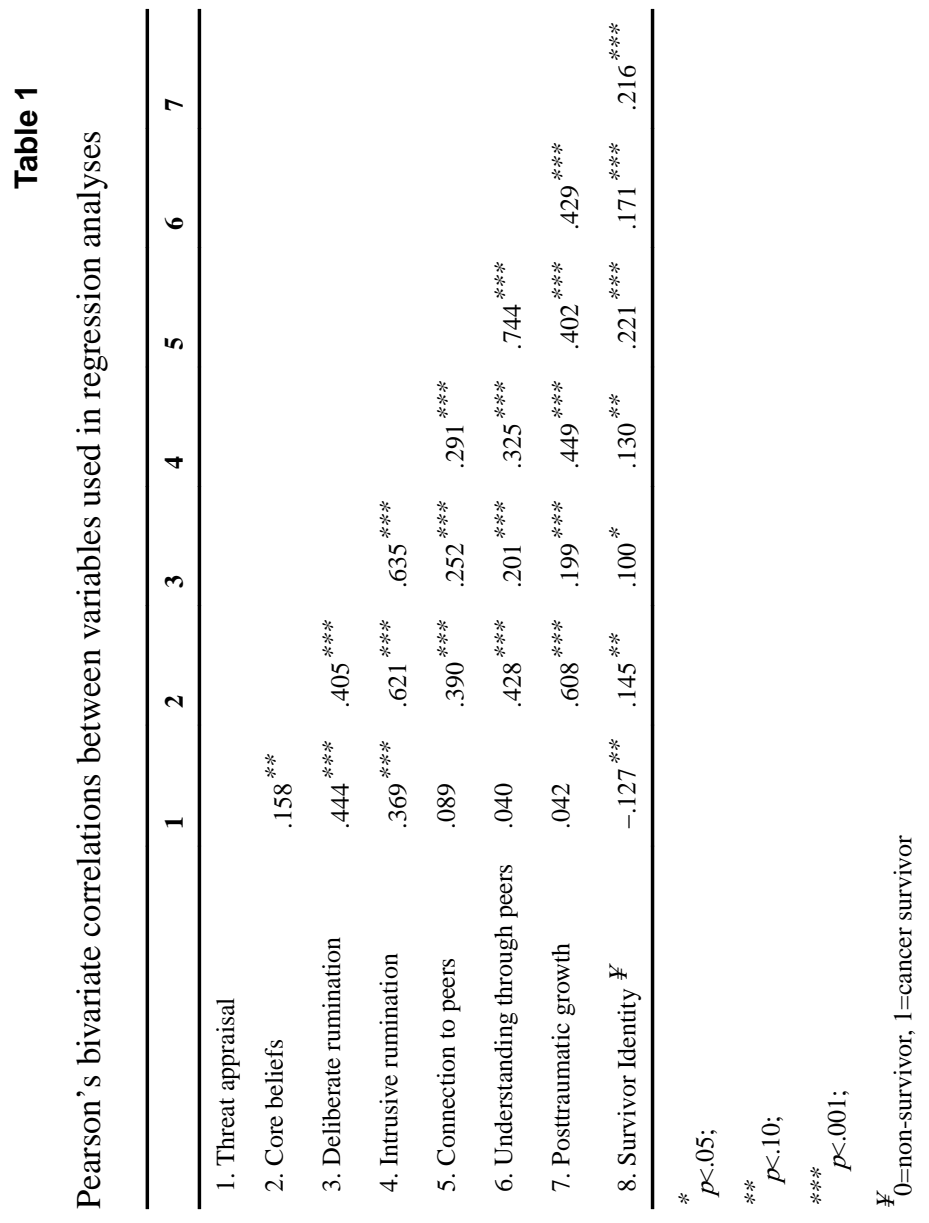




\section{Table 2}

Significant $t$-tests comparing psychosocial variables

\begin{tabular}{llll}
\hline & Survivor identity & & $t$ test $(\mathbf{d f}), \boldsymbol{p}$ \\
\hline & No $\boldsymbol{M}(\mathrm{SD})$ & Yes $\boldsymbol{M}(\mathrm{SD})$ & \\
Threat appraisal & $2.21(\mathrm{SD}=0.98)$ & $1.96(\mathrm{SD}=0.84)$ & $2.81(481), p=.005$ \\
Core beliefs & $1.85(\mathrm{SD}=1.24)$ & $2.23(\mathrm{SD}=1.24)$ & $-3.23(488), p=.001$ \\
Intrusive rumination & $11.28(\mathrm{SD}=7.19)$ & $13.20(\mathrm{SD}=6.69)$ & $-2.91(494), p=.004$ \\
Deliberate rumination & $12.50(\mathrm{SD}=8.26)$ & $14.17(\mathrm{SD}=7.62)$ & $-2.22(491), p=.027$ \\
Understanding through peers & $3.38(\mathrm{SD}=0.89)$ & $3.69(\mathrm{SD}=0.74)$ & $-3.88(500), p=.000$ \\
Connection to peers & $3.54(\mathrm{SD}=0.74)$ & $3.88(\mathrm{SD}=0.67)$ & $-5.07(500), p=.000$ \\
Posttraumatic growth & $46.42(\mathrm{SD}=23.37)$ & $56.80(\mathrm{SD}=20.92)$ & $-4.95(501), p=.000$ \\
\hline
\end{tabular}




\section{Table 3}

Logistic regression model of cancer survivor identity

\begin{tabular}{|c|c|c|c|}
\hline & OR & $95 \% \mathrm{CI}$ & $p$ \\
\hline Age & 0.99 & $0.96,1.03$ & .664 \\
\hline \multirow[t]{2}{*}{ Relationship status ${ }^{a}$} & 0.85 & $0.33,2.16$ & .725 \\
\hline & 0.68 & $0.24,2.55$ & .682 \\
\hline \multirow[t]{3}{*}{ Education status ${ }^{a}$} & 0.94 & $0.34,2.62$ & .904 \\
\hline & 1.32 & $0.48,3.64$ & .597 \\
\hline & 1.32 & $0.47,3.70$ & .603 \\
\hline Time since diagnosis & 1.04 & $0.99,1.09$ & .139 \\
\hline Threat appraisal & 0.59 & $0.45,0.79$ & .000 \\
\hline Core beliefs & 0.94 & $0.73,1.21$ & .652 \\
\hline Deliberate rumination & 1.04 & $1.00,1.08$ & .042 \\
\hline Intrusive rumination & 1.02 & $0.97,1.07$ & .434 \\
\hline Understanding through peers & 1.59 & $1.02,2.49$ & .041 \\
\hline Connection to peers & 0.94 & $0.64,1.39$ & .761 \\
\hline Posttraumatic growth ${ }^{*}$ & 1.01 & $1.00,1.03$ & .052 \\
\hline
\end{tabular}

OR odds ratio, $C I$ confidence interval; records with missing values were listwise deleted; significant relationships in bold font;

borderline significance

${ }^{a}$ Variables entered as categorical variables (relationship status 1 in a relationship, 2 not in a relationship and 3 widowed; education status 1 university, 2 trade or technical college, 3 high school and 4 primary school) 


\section{Table 4}

Comparisons of support group behaviors, ratings of importance of various helpful group experiences, perceptions of mattering to the group and difficulties in relating to the group among women who have had breast cancer and do or do not identify as a survivor ${ }^{a}$

\begin{tabular}{|c|c|c|c|}
\hline \multirow[b]{2}{*}{ Variables } & \multicolumn{3}{|l|}{ Survivor identity } \\
\hline & $\mathrm{No}^{b} M(\mathrm{SD})$ & $\operatorname{Yes}^{c} M(\mathrm{SD})$ & $t$ test $(\mathrm{df}), p$ \\
\hline \multicolumn{4}{|l|}{ Weekly attendance (6 maximum) } \\
\hline Asynchronous discussion board & $2.51(1.80)$ & $2.95(1.92)$ & $0.22(158), p=.140$ \\
\hline Synchronous chat room & $4.39(1.55)$ & $4.38(1.79)$ & $0.27(158), p=.96$ \\
\hline \multicolumn{4}{|l|}{ Amount of posting (continuous) ${ }^{d}$} \\
\hline Asynchronous discussion board & $6.57(6.63)$ & $9.16(8.99)$ & $1.92(125), p=.057$ \\
\hline Synchronous chat room & $128.95(104.56)$ & $163.48(109.88)$ & $1.99(151), p=.048$ \\
\hline How much you mattered to the group $e^{e}$ & $8.63(4.85)$ & $11.05(4.66)$ & $3.15(151), p=.002$ \\
\hline Perceived difficulties relating to the group ${ }^{e}$ & $1.96(2.14)$ & $1.00(1.39)$ & $3.21(144), p=.002$ \\
\hline \multicolumn{4}{|l|}{ Importance of helpful group experiences $e$} \\
\hline Emotional and belonging support & $2.17(1.09)$ & $2.68(1.02)$ & 2.93 (144), $p=.004$ \\
\hline Cognitive growth and information & $2.35(1.13)$ & $2.78(0.92)$ & $2.57(144), p=.01$ \\
\hline Disclosure & $2.50(1.05)$ & $2.72(0.90)$ & $1.40(144), p=.16$ \\
\hline Existential concerns & $1.82(1.05)$ & $2.26(0.95)$ & $2.65(144), p=.009$ \\
\hline Altruism & $2.27(0.89)$ & $2.67(0.81)$ & $2.59(144), p=.01$ \\
\hline \multicolumn{4}{|c|}{ Sample size ranged from 146 to 160 due to incomplete data from some participants (e.g. those who did not attend chat or use the discussion board } \\
\hline \multicolumn{4}{|c|}{${ }^{b}$ Cancer patient, person who has/had cancer, cancer victim } \\
\hline \multicolumn{4}{|l|}{${ }^{c}$ Cancer survivor, sur-thriver, warrior } \\
\hline \multicolumn{4}{|c|}{$\begin{array}{l}\text { dFor ease of interpretation, reported means and analysis are on raw rather than normalized data; results were the same with analysis of normalized } \\
\text { data }\end{array}$} \\
\hline
\end{tabular}

